\title{
Simulation of Parasitic Interconnect Capacitance for Present and Future ICs
}

\author{
Grzegorz Tosik, Zbigniew Lisik, Malgorzata Langer, \\ and Janusz Wozny \\ Institute of Electronics, Technical University of Łódź, \\ 91-924 Lodz, Wolczanska 223 \\ \{Pgrzegorz.tosik, lisikzby, malanger, \\ jwozny\}@p.lodz.pl
}

\begin{abstract}
The performance of modern integrated circuits is often determined by interconnect wiring requirements. Moreover, continuous scaling of VLSI circuits leads to an increase in the influence of interconnects on system performance. It is desired therefore, to calculate accurately its parasitic components, particularly wiring capacitance. In order to recognize which one from the most popular empirical approaches gives the evaluation of the total capacitance that suits to the real capacitance of the interconnect line, the numerical simulations based on the numerical solving of Maxwell equations have been employed.
\end{abstract}

\section{Introduction}

Due to continually shrinking feature sizes, higher clock frequencies, and the simultaneous growth in complexity, the role of interconnections in determining circuit performance is growing in importance. This trend has led to the increasing dominance of interconnect delay over logic propagation delay - even with new metal technologies such as copper or new low-k dielectrics [1]. Additionally, increasingly large chip dimensions result in the longer interconnect lines, which give considerable contributions to the total power dissipation. Since the metallic interconnections are the crucial design issue for current and future generation of IC's, it is increasingly important to compute accurately all its parasitic components, particularly wiring capacitances. Their evaluation is a non-trivial task and it is a subject of many investigations [2],[3],[4]. There are two major approaches to calculate the parasitic capacitance. The first type is to use a numerical simulation, often based on Finite Difference Time Domain or on Finite Element Method [5],[6]. The numerical methods have good accuracy, however are too time-consuming, when applied to the whole integrated circuit. The second approach uses analytic formulations, derived from the equations of electromagnetism. These methods have a sufficient accuracy and a simulation speed, but they can be considered to simulate a few physical configurations, only. Empirical 2- and 3D capacitance models have been reported extensively in the literature. In order to recognize which one from the most popular approaches [7],[8],[9],[10] gives the evaluation of the total capacitance that suits to 
the real capacitance of the interconnect line, the numerical simulations based on the numerical solving of Maxwell equations have been employed. The simulations were performed using the commercial software package OPERA [11], which uses finite element techniques to analyze the electromagnetic problems.

\section{Electrical Models of On-chip Interconnections}

Initially, interconnect has been modeled as a single lumped capacitance in the analysis of the performance of on-chip interconnects. Currently, at low frequency, the lumped RC models are used for high-resistance nets (Fig.1a) and capacitance models are used for less resistive interconnect [8],[12]. To represent the distributed nature of the wire, the interconnect is broken down into $n$ smaller lumped section (Fig.1b). The simulation accuracy increases with increasing $n$. If the signal rise time is too short or the wire is very long, the inductance must also be included and an RLC network (Fig.1c), or the transmission line model (Fig.1d) must be used [13],[14]. The possible representations of interconnect line models include the $\Pi, \mathrm{L}$ and $\mathrm{T}$ networks.

(a)

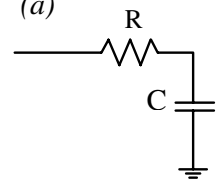

(c)

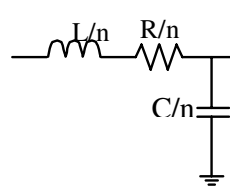

(b)

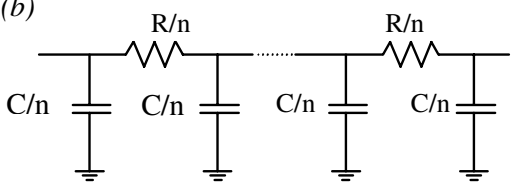

(d)

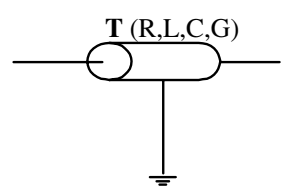

Fig. 1. Models of interconnections. (a) Lumped RC line (b) Distributed RC line. (c) Distributed RLC line (d) Lossy transmission line.(n: number of distributed cells)

\section{Analytical Models of Parasitic Interconnect Capacitance}

An accurate model for the crossover capacitance is essential for estimating the interconnect circuit performance. To get an accurate interconnect capacitance electric field solvers (2D or 3D) should be used. It is, however, so huge task that it would take ages to estimate the capacitance of the whole chip. Therefore, various assumptions and approximations are used to get quick estimates. The empirical formulas, derived from the equations of electromagnetism have the sufficient accuracy and the simulation speed. The simplest equation for interconnect capacitance is given by.

$$
\mathrm{C}=\varepsilon_{\mathrm{o}} \varepsilon_{\mathrm{SiO} 2} \frac{\mathrm{W}}{\mathrm{H}} \mathrm{L}_{\mathrm{Int}}
$$


where $\mathrm{H}$ is the interlayer dielectric (ILD) thickness, $\varepsilon_{\mathrm{SiO} 2}$ is dielectric constant, $\varepsilon_{\mathrm{o}}$ is the permittivity of free space, $\mathrm{W}$ and $\mathrm{L}_{\mathrm{Int}}$ are the width and the length of the interconnect respectively.

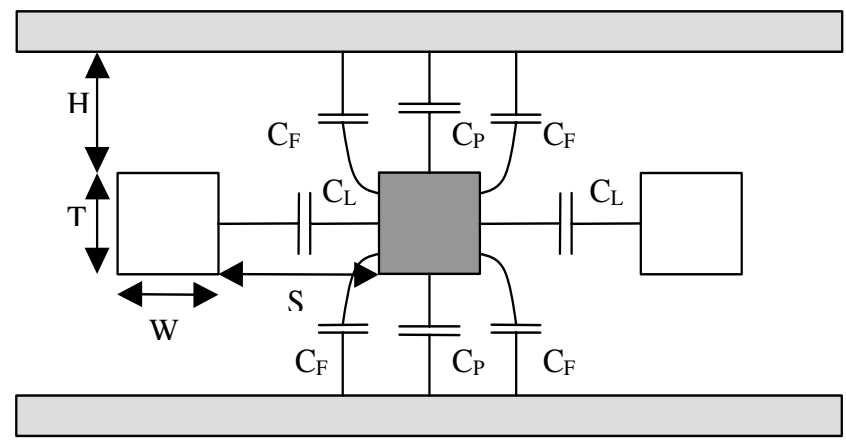

Fig. 2. Interconnect capacitance components

For interconnections in modern integrated circuits a large part of the capacitance comes from lateral coupling between adjacent wires placed on the same metal level and from fringing fields This means that the values of extracted capacitance using simple parallel plate capacitor approximations are extremely inaccurate. In reality, the total interconnect capacitance is a sum of a few different capacitances resulting from the particular design of interconnect system and cannot be treated as the simple plane described by (1). As it is shown in Fig.2, one can distinguish the parallel plate capacitance component, $\mathrm{C}_{\mathrm{P}}$, the fringing field component, $\mathrm{C}_{\mathrm{F}}$, and lateral coupling capacitance component, $\mathrm{C}_{\mathrm{L}}$. It should be noted, that if the neighbor line switches in the opposite direction, the effective lateral capacitance doubles but if the neighbor line switches in the same direction the effective lateral capacitance equals to zero.

There are several approaches to identify the total capacitance $\mathrm{C}_{\text {Total }}$, which use different models to define the components capacitances The most popular of them are presented below. One of the first one was developed by Sarasvat [7]. He considered the structure shown in Fig. 2 and described the component capacitances as follows:

$$
\begin{array}{r}
\mathrm{C}_{\mathrm{P}}=\varepsilon_{\mathrm{ox}} \varepsilon_{\mathrm{o}} \frac{\mathrm{W}}{\mathrm{H}} \\
\mathrm{C}_{\mathrm{L}}=\varepsilon_{\mathrm{ox}} \varepsilon_{\mathrm{o}} \frac{\mathrm{T}}{\mathrm{S}} \\
\mathrm{C}_{\text {Total }}=\mathrm{k}\left(2 \mathrm{C}_{\mathrm{P}}+2 \mathrm{C}_{\mathrm{L}}\right)
\end{array}
$$

where $\mathrm{T}$ - line thickness, $\mathrm{S}$ - distance between two adjacent wires and $\mathrm{k}$ - the factor which takes into account the fringing fields, which value can be calculated using twodimensional analysis of Dang and Shigyo [15].

Another model was presented by Sakurai [8] who derived a simple analytical formula for the total capacitance for symmetrical interlevel dielectric thickness. However, this formula takes into account a basic wire structure with one ground plane only. Because of the symmetrical nature of the interconnect structure considered in 
this work, like is shown in Fig.2 the capacitance between wire and ground $\mathrm{C}_{\text {vertical }}$ and capacitance between adjustment wires $\mathrm{C}_{\text {horizontal }}$ are multiplied by two.

$$
\begin{gathered}
\mathrm{C}_{\text {vertical }}=\varepsilon\left(1.15\left(\frac{\mathrm{W}}{\mathrm{H}}\right)+2.80\left(\frac{\mathrm{T}}{\mathrm{H}}\right)^{0.222}\right) \\
\left.\mathrm{C}_{\text {horizontal }}=\varepsilon\left(0.03\left(\frac{\mathrm{W}}{\mathrm{H}}\right)+0.83\left(\frac{\mathrm{T}}{\mathrm{H}}\right)-0.07\left(\frac{\mathrm{T}}{\mathrm{H}}\right)^{0.222}\right)\left(\frac{\mathrm{S}}{\mathrm{H}}\right)^{-1.34}\right) \\
\mathrm{C}_{\text {Total }}=2 \mathrm{C}_{\text {vertical }}+2 \mathrm{C}_{\text {horizontal }}
\end{gathered}
$$

Chern in [9] presented a more complex crossover model for triple-level metal layer, which was the base for the formulas below:

$$
\begin{gathered}
\mathrm{C}_{\text {vertical }}=\varepsilon\left(\frac{\mathrm{W}}{\mathrm{H}}+1.086\left(1+0.685 \mathrm{e}^{\frac{-\mathrm{T}}{1.343 \mathrm{~S}}}-0.9964 \mathrm{e}^{\frac{-\mathrm{S}}{1.421 \mathrm{H}}}\right)\left(\frac{\mathrm{S}}{\mathrm{S}+2 \mathrm{H}}\right)^{0.0476}\left(\frac{\mathrm{T}}{\mathrm{H}}\right)^{0.337}\right) \\
\mathrm{C}_{\text {horizontal }}=\varepsilon\left(\frac{\mathrm{T}}{\mathrm{S}}\left(1-1.897 \mathrm{e}^{\frac{-\mathrm{H}}{0.31 \mathrm{~S}}-\frac{-\mathrm{T}}{2.474 \mathrm{~S}}}+1.302 \mathrm{e}^{\frac{-\mathrm{H}}{0.082 \mathrm{~S}}}-0.1292 \mathrm{e}^{\frac{-\mathrm{T}}{1.421 \mathrm{~S}}}\right)\right. \\
\left.+1.722\left(1-0.6548 \mathrm{e}^{\frac{-\mathrm{W}}{0.3477 \mathrm{H}}}\right) \mathrm{e}^{\frac{-\mathrm{S}}{0651 \mathrm{H}}}\right) \\
\mathrm{C}_{\text {Total }}=2 \mathrm{C}_{\text {vertical }}+2 \mathrm{C}_{\text {horizontal }}
\end{gathered}
$$

The last formula that will be presented here is taken from Wong work [10]. According to his model, the interconnect capacitance is described as:

$$
\begin{gathered}
\mathrm{C}_{\text {vertical }}=\varepsilon\left(\frac{\mathrm{W}}{\mathrm{H}}+2.217\left(\frac{\mathrm{S}}{\mathrm{S}+0702 \mathrm{H}}\right)^{2.193}+1.17\left(\frac{\mathrm{S}}{\mathrm{S}+1.510 \mathrm{H}}\right)^{0.7642}\left(\frac{\mathrm{T}}{\mathrm{T}+4.532 \mathrm{H}}\right)^{0.1204}\right. \\
\mathrm{C}_{\text {horizontal }}=\varepsilon\left(1.412 \frac{\mathrm{T}}{\mathrm{S}} \exp \left(\frac{-4 \mathrm{~S}}{\mathrm{~S}+8.014 \mathrm{H}}\right)+2.3704\left(\frac{\mathrm{W}}{\mathrm{W}+0.3078 \mathrm{~S}}\right)^{0.25724}\right. \\
\left.\left(\frac{\mathrm{H}}{\mathrm{H}+8.961 \mathrm{~S}}\right)^{0.7571} \exp \left(\frac{-2 \mathrm{~S}}{\mathrm{~S}+6 \mathrm{H}}\right)\right) \\
\mathrm{C}_{\text {Total }}=2 \mathrm{C}_{\text {vertical }}+2 \mathrm{C}_{\text {horizontal }}
\end{gathered}
$$

\section{Numerical Model of Parasitic Interconnect Capacitance}

In order to recognize which from the above formulas gives the evaluation of the total capacitance that suits to the real capacitance of the interconnect line, the numerical simulations based on the numerical solving of Maxwell equations have been employed. The simulations were performed using the Vector Field commercial software package OPERA [11], which uses finite element method to analyze the electromagnetic problems in $2 \mathrm{D}$ domain. This method divides the studied structure into sub-domains. Then, it is possible, with this tool, to fit any polygonal shape by choosing element shapes and sizes. 


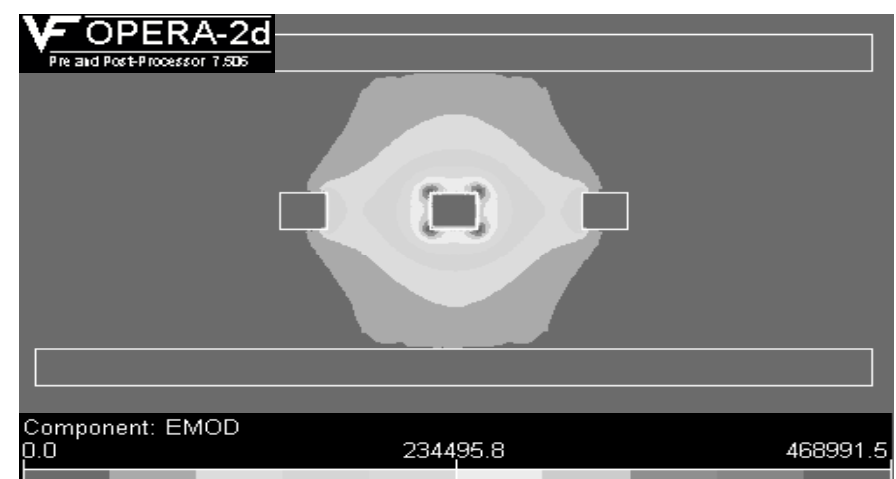

Fig. 3. Interconnect structure used in numerical capacitance calculation

The test interconnect structure is shown schematically in Fig.3. It contains three wires sandwiched between a two ground planes. Three copper lines are imbedded in a dielectric matrix (with the permittivity dependent on technology). The electric potentials and the currents have been fixed to arbitrary values. The cross-section dimension of investigated structure has been changed according to the technology. Typical views of the field distributions are represented in Figure 3. The shades give the intensity of the electric potential. This gives qualitative indications on the intensity of the coupling strengths. The software reports the total charge on the strip in Coulombs per meter. Since the total charge equals capacitance times voltage $(C V=Q)$ then we can interpret the results from the software directly as capacitance per meter.

\section{Comparison Between Analytical and Numerical Models}

The total capacitance was calculated numerically and using four formulas mentioned above. Such a procedure has been repeated for several structures fabricated in 0.13 , 0.10 and $0.05 \mu \mathrm{m}$ CMOS processes, which design parameters are collected in Table.1.

\begin{tabular}{|lcccccccc|}
\hline \multicolumn{7}{c|}{ Table 1. Cross-section interconnect dimensions } & & \\
\hline \hline Technology & Layer & $\mathrm{w}$ & $\mathrm{t}$ & $\mathrm{s}$ & $\mathrm{h}$ & $\mathrm{Eps}$ & $\mathrm{V}$ \\
\hline \multirow{4}{*}{130} & & {$[\mathrm{~m}]$} & {$[\mathrm{m}]$} & {$[\mathrm{m}]$} & {$[\mathrm{m}]$} & - & {$[\mathrm{V}]$} \\
& 1 & $1.46 \mathrm{E}-07$ & $1.08 \mathrm{E}-07$ & $1.54 \mathrm{E}-07$ & $3.55 \mathrm{E}-07$ & 2.00 & 1.20 \\
& 6 & $2.35 \mathrm{E}-06$ & $2.00 \mathrm{E}-06$ & $2.53 \mathrm{E}-06$ & $6.67 \mathrm{E}-06$ & 2.00 & 1.20 \\
100 & 4 & $1.18 \mathrm{E}-07$ & $2.04 \mathrm{E}-07$ & $1.22 \mathrm{E}-07$ & $3.55 \mathrm{E}-07$ & 1.50 & 0.90 \\
& 7 & $2.22 \mathrm{E}-06$ & $2.00 \mathrm{E}-06$ & $4.98 \mathrm{E}-06$ & $6.67 \mathrm{E}-06$ & 1.50 & 0.90 \\
50 & 7 & $1.20 \mathrm{E}-06$ & $1.50 \mathrm{E}-06$ & $1.20 \mathrm{E}-06$ & $9.00 \mathrm{E}-07$ & 1.50 & 0.60 \\
& 9 & $2.00 \mathrm{E}-06$ & $2.50 \mathrm{E}-06$ & $2.00 \mathrm{E}-06$ & $1.40 \mathrm{E}-06$ & 1.50 & 0.60 \\
\hline \hline
\end{tabular}


The discrepancies between the total capacitance evaluation by means of analytical formulas and the OPERA simulations are shown in Table.2. The error used as the discrepancy measure is defined as follows:

$$
\text { Error }=\frac{\text { Analytical calculation }- \text { Numerical calculation }}{\text { Numerical calculation }} 100 \% .
$$

Table 2. Error table of the analytical formulas compared with numerical simulator

\begin{tabular}{ccccccccccc}
\hline \hline Tech. & Layer & OPERA & Mod.1 & error & Mod.2 & error & Mod.3 & error & Mod.4 & error \\
\hline \multirow{2}{*}{130} & 1 & $7.36 \mathrm{E}-14$ & $7.84 \mathrm{E}-14$ & $7 \%$ & $8.26 \mathrm{E}-14$ & $12 \%$ & $1.16 \mathrm{E}-13$ & $58 \%$ & $3.94 \mathrm{E}-14$ & $-46 \%$ \\
& 6 & $7.39 \mathrm{E}-14$ & $7.88 \mathrm{E}-14$ & $7 \%$ & $8.46 \mathrm{E}-14$ & $14 \%$ & $1.17 \mathrm{E}-13$ & $58 \%$ & $4.05 \mathrm{E}-14$ & $-45 \%$ \\
\multirow{2}{*}{100} & 4 & $7.63 \mathrm{E}-14$ & $8.07 \mathrm{E}-14$ & $6 \%$ & $9.19 \mathrm{E}-14$ & $20 \%$ & $1.23 \mathrm{E}-13$ & $61 \%$ & $5.32 \mathrm{E}-14$ & $-30 \%$ \\
& 7 & $4.37 \mathrm{E}-14$ & $4.56 \mathrm{E}-14$ & $4 \%$ & $5.23 \mathrm{E}-14$ & $20 \%$ & $7.51 \mathrm{E}-14$ & $72 \%$ & $1.95 \mathrm{E}-14$ & $-55 \%$ \\
& 7 & $8.61 \mathrm{E}-14$ & $9.22 \mathrm{E}-14$ & $7 \%$ & $1.06 \mathrm{E}-13$ & $23 \%$ & $1.48 \mathrm{E}-13$ & $72 \%$ & $6.86 \mathrm{E}-14$ & $-20 \%$ \\
50 & 9 & $8.93 \mathrm{E}-14$ & $9.39 \mathrm{E}-14$ & $5 \%$ & $1.09 \mathrm{E}-13$ & $22 \%$ & $1.52 \mathrm{E}-13$ & $70 \%$ & $7.11 \mathrm{E}-14$ & $-20 \%$ \\
\hline \hline
\end{tabular}

where Mod.1 is the Chern [9] formula, Mod.2 is the Wong [10] formula and Mod.3 and Mod.4 are the Sakurai [8] and Sarasvat [7] approaches respectively. Based on this comparison one can judge that the empirical Chern's formula that error value is less then $8 \%$ over a wide range of interconnect parameters can be treated as the more realistic one. It should be noted that the valid range of interconnect dimensions for this formula is [9]:

$$
0.3 \leq \frac{\mathrm{W}}{\mathrm{H}} \geq 10 ; \quad 0.3 \leq \frac{\mathrm{H}}{\mathrm{T}} \geq 10
$$

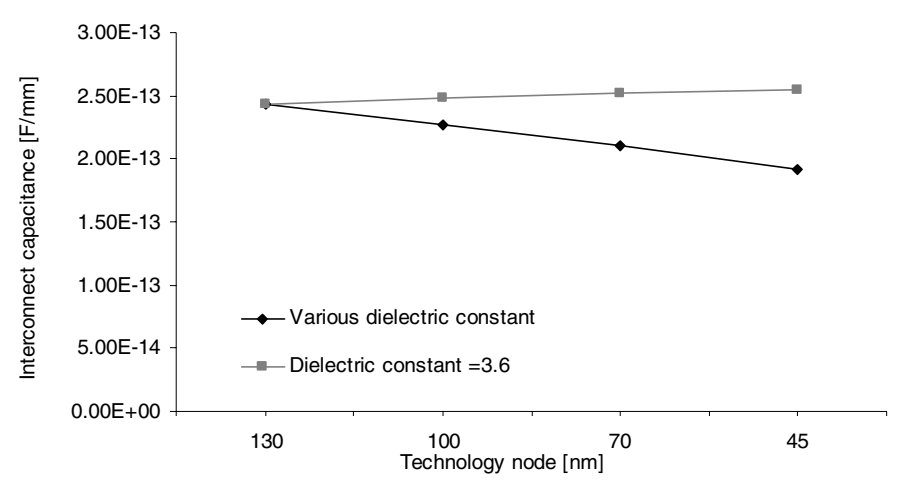

Fig. 4. Global interconnects capacitance versus technology node

Fig. 4 shows the wiring capacitance per unit length $\mathrm{C}_{\mathrm{o}}$ calculated by Chern's formula as a function of technology nodes. The calculations have been done for global interconnect with minimum cross-section dimensions (minimum wire pitch). It can be 
noticed that the interconnect capacitance calculated for the present value of dielectric constant 3.6 remains almost the same, while the capacitance calculated for the values predicted by ITRS [1] tends to lessen.

\section{Conclusion}

The crossover parasitic interconnect capacitance is essential for estimating the interconnect circuit performance. To get an accurate interconnect capacitance electric field solvers should be used. Since it is too time-consuming, the empirical approximations derived from the equations of electromagnetism are used. Unfortunately these methods can be considered to simulate a few physical configurations, only. In order to recognize which from the most popular approaches gives the evaluation of the total capacitance that suits to the real capacitance of the considered interconnect structure (Fig.2), the numerical simulations based on the numerical solving of Maxwell equations have been employed. The presented comparison showed that only for the Chern formula the error value is less then $7 \%$ over a wide range of ITRS parameters. Therefore one can conclude that the Chern formula is the most realistic one for the considered interconnect structure.

\section{References}

[1] The International Technology Roadmap for Semiconductors 2001, http ://public.itrs.net

[2] A.Ruehli P.Brennan "Capacitance Models for Integrated Circuit Metalization Wires "Journal of Solid-State Integrated Circuits Vol.10 No.6 pp.530-536 1975.

[3] M.Lee "A Multilevel Parasitic Interconnect Capacitance Modeling and Extraction for Reliable VLSI On-Chip Clock Delay Evaluation" Journal of Solid-State Integrated Circuits Vol.33 No.4 pp.657-661 1998.

[4] E.Barke "Line-to-Ground Capacitance Calculation for VLSI: A Comparison" IEEE Transaction of Computer Added Design Vol.7 No.2 pp.295-298 1988

[5] J.C.Chen B.McGaughy D.Sylvester C.Hu "An On-Chip Atto-Farad Interconnect ChargeBased Capacitance Measurement Technique" IEEE Tech. Dig. Int. Electron device Meeting 1996.

[6] O.P.Jensen "Calculating Wire Capacitance in Integrated Circuits" IEEE Circuits and Devices No.3 pp.36-40 1994.

[7] K.C.Saraswat F.Mohammadi Effect of Scaling of Interconnections on the Time Delay of VLSi Circuits Journal of Solid-State Circuits Vol.17 No.2 pp.275-280, 1982.

[8] T. Sakurai K. Tamaru Simple Formulas for Two- and Three -Dimensional Capacitances IEEE Tran. On Electron Devices Vol.30 No.2 pp.183-185, 1983.

[9] J.H. Chern, J. Huang, L. Arledge, P.C. Li, and P. Yang, Multilevel metal capacitance models for CAD design synthesis systems, IEEE Electron Device Letters, Vol.13, pp. 3234, 1992.

[10] Shyh-Chyi Wong T.G.Y.Lee D.J.Ma CH.J.Chao An Empirical Three-Dimensional Crossover Capacitance Model for Multilevel Interconnect VLSI Circuits IEEE Trans. Semiconductor Manufacxturing Vol.13 No.2 pp.219-223, 2000.

[11] Vector Fields http://www.vectorfields.com/op2d 
[12] J.Rubinstein P.Penfield M.A.Horowitz "Signal Delay in RC Tree Networks" IEEE Transactions on Computer-Aided Design Vol.2 No.3 pp.202-211 1983

[13] J.A.Davis J.D.Meindl "Compact Distributed RLC Interconnect Models, Part II- Coupled line transient expressions and peak crosstalk in multilevel networks" IEEE Transactions on Electron Devices Vol.47 No.11 pp.2078-2087 2000.

[14] Y.I.Ismail E.G.Friedman "Equivalent Elmore Delay for RLC Trees“ IEEE Transaction on Computer-Aided Design of Integrated Circuits and systems Vol.19 No.1 Pp83-96 Jan 2000.

[15] R.L Dang N.Shigyo A two-dimensional simulations of LSI interconnect capacitance IEEE Electron Device Lett. EDL-2 pp.196-197, Aug 1981. 